\title{
Is the metabolic syndrome caused by a high fructose, and relatively low fat, low cholesterol diet?
}

\author{
Stephanie Seneff', Glyn Wainwright², Luca Mascitelli³
}

1Department of Electrical Engineering and Computer Science, MIT, Cambridge, MA, USA 2Independent Reader of Research, Leeds, United Kingdom

${ }^{3}$ Medical Service, Comando Brigata Alpina "Julia”, Udine, Italy

Submitted: 5 July 2010

Accepted: 23 August 2010

Arch Med Sci 2011; 7, 1: 8-20

DOI: 10.5114/aoms.2011.20598

Copyright (c) 2011 Termedia \& Banach

\section{Abstract}

The metabolic syndrome (MetS) is manifested by a lipid triad which includes elevated serum triglycerides, small LDL particles, and low high-density lipoprotein (HDL) cholesterol, by central obesity (central adiposity), insulin resistance, glucose intolerance and elevated blood pressure, and it is associated with an increased risk of type 2 diabetes and coronary heart disease. We have developed a new hypothesis regarding MetS as a consequence of a high intake in carbohydrates and food with a high glycemic index, particularly fructose, and relatively low intake of cholesterol and saturated fat. We support our arguments through animal studies which have shown that exposure of the liver to increased quantities of fructose leads to rapid stimulation of lipogenesis and accumulation of triglycerides. The adipocytes store triglycerides in lipid droplets, leading to adipocyte hypertrophy. Adipocyte hypertrophy is associated with macrophage accumulation in adipose tissue. An important modulator of obesity-associated macrophage responses in white adipose tissue is the death of adipocytes. Excess exposure to fructose intake determines the liver to metabolize high doses of fructose, producing increased levels of fructose end products, like glyceraldehyde and dihydroxyacetone phosphate, that can converge with the glycolytic pathway. Fructose also leads to increased levels of advanced glycation end products. The macrophages exposed to advanced glycation end products become dysfunctional and, on entry into the artery wall, contribute to plaque formation and thrombosis.

Key words: cholesterol, fructose, metabolic syndrome.

\section{Introduction}

The metabolic syndrome (MetS) is a term that has been used to encapsulate a cluster of metabolic risk factors associated with insulin resistance and heart disease. It has become formalized into slightly different thresholds by the Adult Treatment Panel III (ATP III), the American Heart Association and the International Diabetes Federation, but all agree on the following specific criteria: abdominal obesity defined in terms of large waist girth, high fasting blood glucose, low serum HDL-C, high serum triglycerides, and elevated blood pressure [1]. Typically, the definition requires above-threshold measures on at least three of the five criteria.

Several authors have written detailed reviews of the physiological profile associated with MetS [1-4]. However to our knowledge, no one has thus far attempted to provide a comprehensive unifying theory that explains

\author{
Corresponding author: \\ Luca Mascitelli, MD \\ Medical Service \\ Comando Brigata Alpina "Julia" \\ 8 Via S. Agostino \\ Udine 33100 , Italy \\ Phone: +390432584044 \\ Fax: +390432584053 \\ E-mail: lumasci@libero.it
}




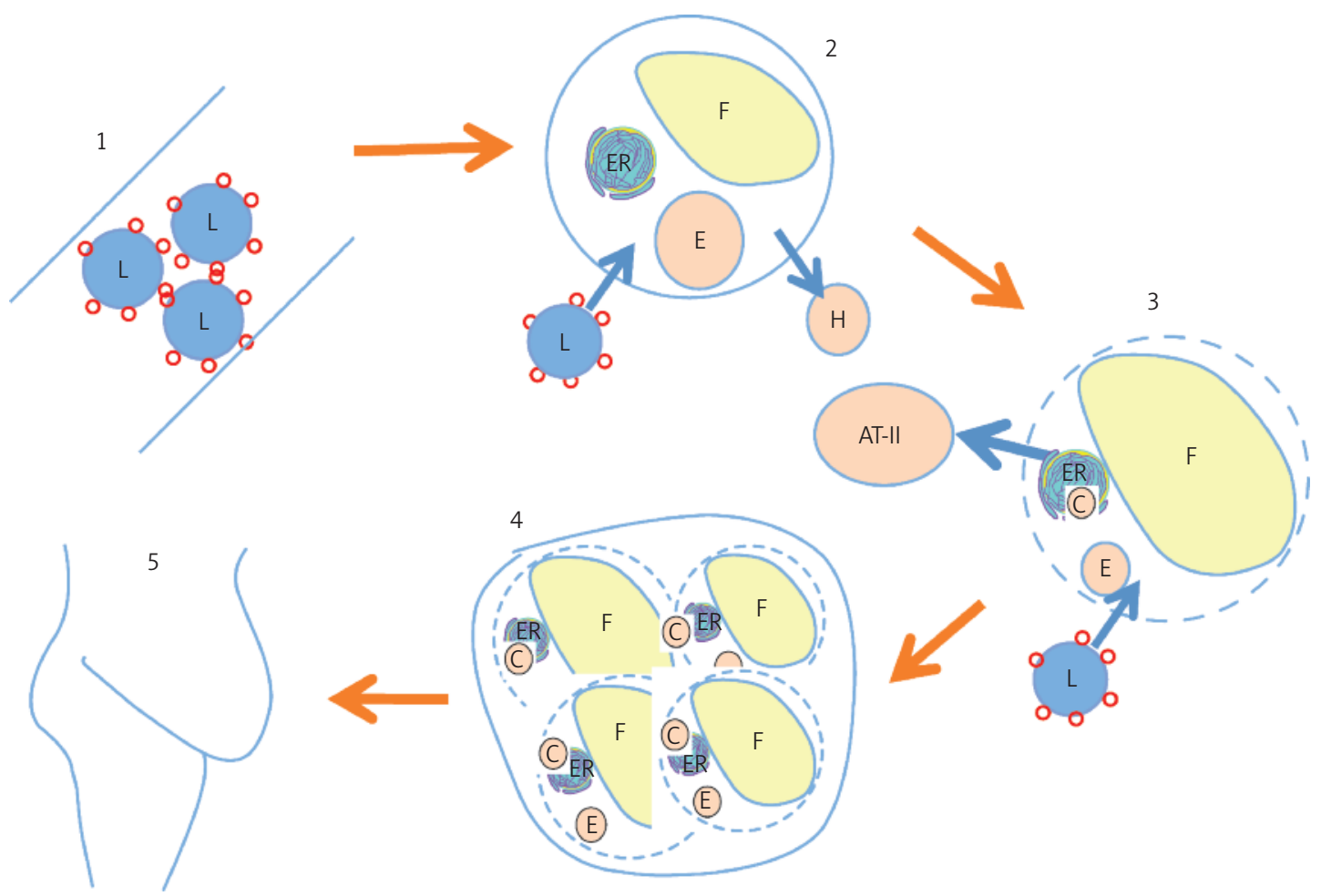

Figure 1. Block diagram of proposed sequence of events leading to MetS. (1) Serum LDL (L) becomes glycated due to exposure to glucose and fructose. (2) Adipocytes depend upon apoE (E) to scavenge glycated LDL-C and transport it into HDL-A1 (H). (3) ApoE becomes damaged and adipocytes accumulate lipid droplets (F) and excess cholesterol (C) in their endoplasmic reticulum (ER). Meanwhile, plasma membrane becomes cholesterol-depleted. Stressed adipocytes release angiotensin-II (AT-II) which leads to sodium-hoarding and hypertension. (4) Macrophages enter adipose tissue to engulf cell debris from accumulating dead adipocytes, forming multi-nucleated giant cells. (5) Due to insufficient HDL-C, fatty deposits accumulate ectopically to buffer cholesterol supplies to the major organs

the disease progression and accounts for the diverse physiological pathologies associated with MetS. Several studies have shown predictive power of MetS beyond the effects of the individual measures [5-7], although others argue that it is not a useful diagnostic metric beyond the component measures [3]. Nonetheless, MetS carries a substantially increased risk of atherosclerosis and subsequent cardiovascular disease, and is associated with an increased likelihood of a shortened life span [8]. Over the last several decades, medical advice, particularly in the United States, has emphasized the concept that a low-fat diet is a healthy diet, and this has likely led to a shift towards an increased dietary intake in carbohydrates. However, recent studies have demonstrated that a low-carbohydrate diet leads to improvements on a number of measures related to heart disease and diabetes risks $[9,10]$. As a consequence of these contradictions, considerable confusion exists as to what constitutes healthy eating. A case in point is the recent review article on recommended lifestyle changes to improve cardiovascular risk factors, which recommended reduced fat intake twice and recommended reduced carbohydrate intake twice, while suggesting that protein intake should remain under $20 \%$ of total calories [11].

Fortunately, substantial recent research has uncovered a large amount of detailed information about the intricate biological processes concerned with cholesterol, fatty acid, and glucose homeostasis. Of particular note for our purposes is the low serum HDL-C associated with MetS. In a study of 7-year-old children, it was shown that already by this young age there existed a correlation between low serum HDL-C and an overabundance of small LDL particles [12]. This effect preceded any evidence of MetS, suggesting that this feature is indeed an early indicator of the syndrome. It has been further observed that the HDL is also depleted in cholesterol. In fact, there is an inverse relationship between the degree of insulin resistance and the amount of cholesterol contained in $\operatorname{HDL}[13,14]$, a highly significant fact that motivates our arguments.

\section{The big picture}

In this paper, we developed a theory accounting for all the features of MetS, which involves 
a cascade of events brought on by gross dietary imbalances. We argue that this syndrome has reached epidemic proportions due to misguided advice regarding a "healthy" lifestyle, leading to reduced dietary intake of fats and cholesterol and excessive sun avoidance. The increasingly widespread availability of highly processed foods, particularly the practice of substituting fructose for glucose as a sweetener due to economic considerations, has been an equally damaging contributing factor. Calcium and vitamin D deficiency play a role as well.

It is hypothesized (Fig. 1) that the condition may begin with liver dysfunction in converting fructose to fat and struggling to produce enough cholesterol to buffer the synthesized fat. The liver then falls behind in its role in processing glucose, leaving a large percentage of this burden to adipocytes. Adipocytes are also tasked with recycling the cholesterol extracted from serum LDL that has become damaged due to excess exposure to glucose and fructose. Over time, the adipocytes' protein synthesizing machinery becomes defective. The adipocytes become engorged with fat stores, and are unable to maintain a healthy plasma membrane (PM). Their last act is to release cytokines calling in macrophages in anticipation of their imminent disintegration. Eventually, the adipose tissue becomes riddled with giant cells, multinucleated macrophages attempting to protect the contents of dysfunctional adipocytes from further glycation, fructation, and oxidation.

The stressed adipocytes and their accumulating macrophage assistants release hormones and peptides that trigger a cascade of events with the goal of assuring that the tissues are adequately supplied with fat and cholesterol, a feat that they are nonetheless unable to accomplish in the face of continuing chronic exposure to excess fructose and glucose. PM of active cells in the pancreas, the liver, the heart, the kidneys, the skeletal muscles, and the blood serum organelles all become deficient in cholesterol and therefore highly vulnerable to attack by sugars and oxygen. The body accumulates ectopic deposits of fats and cholesterol in order to provide these vital nutrients when necessary. Glucose and fructose accumulate in the bloodstream in ever greater amounts due to the damage accrued by the $\beta$ cells in the pancreas and the reduced ability of the heart and skeletal muscles to utilize sugars as fuel due to their compromised PM.

\section{Liver dysfunction associated with high fructose diet}

Several experiments have been performed, on both humans and animal models, that clearly show that fructose is highly damaging to health [15-18].
In experiments with rats, fructose has been shown to induce hypertension [16], increased arterial stiffening due to damage to collagen, and decreased cardiac output [17]. In a recent clinical trial [19], it was shown that fructose overconsumption in humans leads to dyslipidemia and ectopic lipid deposition, along with increased hepatic insulin resistance. In a crossover design, both hepatic lipids and VLDL triacylglycerides increased by over $75 \%$ following a short-term highfructose diet. Lipids stored in skeletal muscle cells also increased significantly. Furthermore, wholebody insulin sensitivity decreased by $27 \%$. In another study comparing 20 type 2 diabetics on oral medication with normal controls, it was found that, while the ratio of Haemoglobin-A1c (haemoglobin damaged by glucose exposure) between the diabetics and the controls was less than 2.0 (9.3 vs. 5.4), the ratio of serum fructosamine levels differed by a factor of 7 (1127 vs. 170) [20]. Controlled dietary experiments conducted with baboons and with hamsters offer compelling evidence for the damage that chronic fructose exposure does to the liver.

\section{Dietary experiments with baboons}

An experiment conducted in the mid 1970s with baboons [21] involved feeding them a synthetic zero-cholesterol diet. Four test groups were fed different proportions of starch, glucose, fructose, and sucrose, supplemented with appropriate amounts of other dietary requirements besides cholesterol. All of the test groups exhibited an increase in serum triglycerides ranging from $37 \%$ in the starch and glucose-fed groups to $65 \%$ in the fructose-fed group. Liver triglyceride and cholesterol were elevated in all groups. An analysis of bile acid composition indicated a reduced conversion of cholesterol to bile acids. All tested animals developed fatty streaks in the aorta, something that is unusual for this species. The severity of fatty streak development was greatest for the fructosefed group.

\section{Dietary experiments with hamsters}

Hamsters are valuable as a model for humans due to their similar lipoprotein management system, in contrast to rats and mice. A study involving both in vivo and in vitro investigations of the effects of fructose on fat and cholesterol metabolism in hamsters [22] provides great insight into the source of liver dysfunction in the metabolic syndrome. Hamsters were fed a high fructose diet for a two-week period, at the end of which measures were made of blood serum levels of a number of indicators of MetS. A more than 3-fold increase was found in the amount of triglyceride in 
the serum of the fructose-fed hamsters compared to controls, as well as a significant elevation of free fatty acids (FFA) and insulin. A reduction in tyrosine phosphorylation of insulin receptor in the hepatic cells indicated insulin resistance.

After the two-week period, hepatic cells were isolated and grown in medium. Measures were made of both internal and secreted levels of cholesterol, triglycerides, and apolipoprotein B (apoB). It became clear that fructose-fed hamsters retained significantly more fat stores in the liver, with a concurrent $50 \%$ increase in internal storage of free cholesterol. There was a 4.6-fold increase in the amount of VLDL apoB secreted, along with a significantly enhanced intracellular stability of apoB. Most significant for our purposes, the amount of free cholesterol released by fructose-fed hamsters was reduced by more than $50 \%$, and the released VLDL particles contained significantly less cholesteryl ester relative to their triglyceride levels; i.e., these particles were depleted in cholesterol. Overall, it seems clear that the hamsters were forced to retain free cholesterol within the liver to buffer the fatty acids synthesized from the fructose. This prevented them from releasing sufficient cholesterol to the serum, which, over time, leads to insufficient cholesterol supplied to the tissues. At the same time, their insulin resistance was conceivably due to the fact that they were compelled to synthesize cholesterol, and insulin suppresses cholesterol synthesis [23].

\section{A novel theory for the metabolic syndrome}

In our view, MetS arises out of a dietary imbalance with an overabundance of refined, highglycemic index carbohydrates, most notably, fructose, and a relative dietary deficiency in cholesterol. A recently published paper [24] demonstrated an intriguing relationship between fasting glucose level and cholesterol metabolism. For people exhibiting insulin resistance, cholesterol synthesis was upregulated and cholesterol absorption was downregulated, independent of obesity level. This suggests that a dietary deficiency in cholesterol, or an impaired ability to absorb it, may be associated with insulin resistance.

A study of the relationship between dietary cholesterol and serum cholesterol in rats, undertaken in the mid 1970s, uncovered some surprising results [25]. Most notably, a cholesterolfree diet resulted in greater accumulation of cholesterol in adipose tissue than a diet enriched with 0.05 or $0.1 \%$ cholesterol. Furthermore, even up to a $5 \%$ cholesterol diet, serum cholesterol concentration was inversely related to dietary supply after a two-month interval. These authors also demonstrated compellingly that the amount of cholesterol stored in adipose tissue is proportional to the amount of triglycerides stored. Leptin-deficient (ob/ob) mice, (a model of type 2 diabetes with relatively mild hyperglycaemia and obesity) had five times the adipose mass of controls, and 1.2- to 2-fold differences in fat cell diameter, yet the cholesterol to triglyceride ratios were identical between the two groups.

Fructose is especially damaging because it is highly reactive as a reducing agent, and the liver must remove it aggressively from the blood serum to prevent it from damaging serum lipids and proteins via fructation [26]. With a highcarbohydrate, low-fat diet, postprandial fructose and glucose enter the bloodstream very rapidly due both to the abundance of refined carbohydrates and to the lack of buffering in the gut by dietary fats. The tissues are reluctant to utilize fructose as fuel, likely because it is ten times as reactive as a reducing agent as glucose [26].

An excess of fructose and glucose in the bloodstream causes extensive glycation damage to vulnerable proteins [27, 28]. A glycated (whether fructated or glucated) protein is typically both impaired in its function and more susceptible to oxidation damage. It is also resistant to degradation through lysosomal breakdown. Over time, a collection of glycated protein debris accumulates in the blood serum and along arterial walls. These damaged proteins are referred to collectively as advanced glycation end products (AGEs) [29], and they play a critical role in aging, in atherosclerosis, and in the health issues associated with long-term diabetes. Collagen, haemoglobin, LDL, and albumen are all susceptible to AGE damage. In particular, the lysine residues of apo-B in LDL are susceptible to glycation and, once they are glycated, $\mathrm{LDL}$ is only poorly recognized by lipoprotein receptors and scavenger receptors [30]. A schematic of the receptor-mediated uptake of cholesterol and fatty acids from LDL is illustrated in Figure 2 and an illustration of impairment of this process through glycation is given in Figure 3.

Glycation of LDL also causes it to be more susceptible to oxidation damage. A study of the potential effects of lipoprotein glycation on the oxidation of contained cholesteryl esters was conducted by Ravandi et al. [31]. In controlled in vitro experiments, the presence of a synthesized glucosylated lipid, phosphatidylethanolamine (Glc PtdEtn) resulted in a 4 - to 5 -fold increase in the generation of oxidation products such as hydroperoxides and aldehydes. Furthermore, when this AGE product was included in the LDL lipid monolayer, it resulted in rapid loss of cholesteryl esters from the interior. The authors concluded that the presence of glucosylated phospholipids in the membrane may promote oxidation of both the membrane phospholipids and cholesteryl esters in the interior of the particle. 


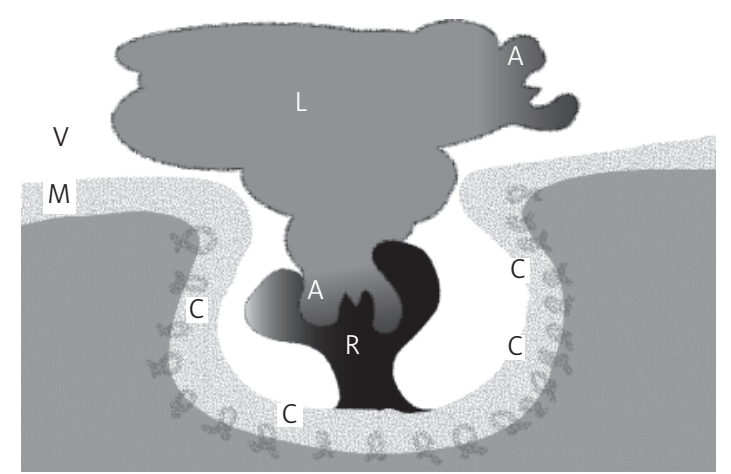

Figure 2. Endocytosis of normal LDL. This schematic represents the normal binding of the apolipoprotein (A) and absorption of an LDL particle (L), which has attached to the receptor $(R)$. The activated receptor has caused the formation of a caveolus for absorption from the blood vessel (V) by endocytosis through the cell membrane $(M)$. These functional membrane changes are facilitated by cholesterol and caveolin enrichments to the lipid raft area (C)

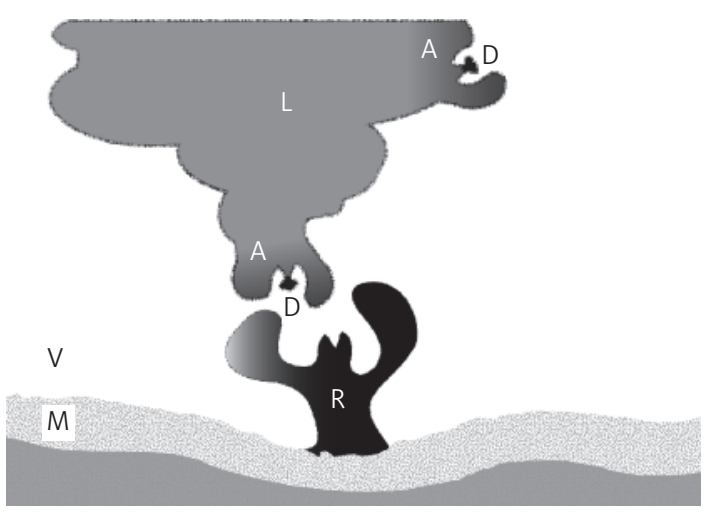

Figure 3. Failed Endocytosis of glycated LDL. In this schematic the lysine in apoB or $E(A)$ has become damaged by glycation (D). Consequently, the receptor $(R)$ is unable to recognize the $L D L$ particle (L). The cell endocytosis via the cell membrane (M) does not occur. The damaged LDL will circulate until a less discriminating scavenger receptor in an adipose cell takes it up for recycling

The liver releases its synthesized fats and cholesterol as VLDL particles, which deliver fat, cholesterol, and antioxidants to all the tissues, while becoming steadily smaller as they migrate through stages of IDL, LDL, and, finally, VLDL remnants, also known as small dense LDL: small lipoprotein shells with minimal content but damaged by exposure to glucose, fructose, and oxygen. The liver is responsible for recycling these remnants through bile excretion to aid in the digestion of fats (and to be reconstituted as free cholesterol in the membrane and esterified cholesterol in the contents of the chylomicron). When there are relatively few fats in the diet, less bile is needed, and the liver, being burdened by fructose and glucose metabolism, falls behind on the task of providing cholesterol to the bile salts.

Meanwhile, the task of cleaning up damaged VLDL remnants is delegated to the adipocytes. In particular, they synthesize substantial amounts of apoE to reconstitute damaged cholesterol and orchestrate its transport to PM so that it can be utilized both by the adipocyte itself and by many other cells in the body (after it is taken up by HDL particles in the bloodstream). Over time, the adipocyte accumulates AGE products due to its chronic exposure to both glucose and damaged VLDL remnants. ApoE is especially susceptible to AGE damage [34], and, eventually, it can no longer function. This leads to the accumulation of excess free cholesterol within the adipocyte, ironically while it is suffering from cholesterol deficiency in its outer wall. The adipocytes are required to store the excess cholesterol. However, the increased size requires a corresponding expansion in the surface area. Without sufficient cholesterol in the PM, the cell becomes first permeable to outward sodium leaks 
but ultimately unable to keep calcium out, at which point the cell literally disintegrates.

Macrophages are responsible for engulfing lipids that are exposed to the interstitial spaces, so they rush in to clean up the cell debris left behind by dead adipocytes. In fact, so-called giant cells accumulate in unhealthy adipose tissue: a single cell encasing multiple cell nuclei and lipid droplets [35]. Such a cell is likely taking advantage of the same principle as that used by the chylomicron. A single PM surrounding multiple cell nuclei requires significantly less cholesterol to protect the contents from damage than would several individual cell walls. The macrophages secrete various inflammatory agents such as interleukin (IL)- 6 and tumour necrosis factor- $\alpha$ (TNF- $\alpha$ ).

Over time, more and more adipocytes swell in size to the point of cell death to accommodate cholesterol that they cannot discharge, and the subcutaneous adipocytes become increasingly unable to deliver refurbished cholesterol to the tissues. Cholesterol deficiency becomes a problem for cells throughout the body, with dire consequences. One consequence will be the increased susceptibility of the fats in cell membranes to oxidation [36]. This problem can be partially ameliorated through the accumulation of fat storage in non-adipocyte cells in and among the viscera, including the heart, i.e., ectopic fat. Epicardial fatty deposits (and ectopic fat in general) serve as a private source of fats and cholesterol to replenish supply to repair damaged membranes when blood serum levels are insufficient.

Initially, it is only the liver that is resistant to insulin, but the skeletal muscles and adipocytes also show signs of insulin resistance as they become exposed to accumulated AGE damage. The resulting excess of glucose in the blood leads to a sharply increased demand for insulin, which imposes excess energy requirements on the $\beta$ cells in the pancreas, leaving them susceptible to glycation and oxidation damage as well. $\beta$ cells require glucose, calcium, fats, vitamin D and cholesterol to all be present at adequate levels in the cytoplasm before they will release insulin. Due to deficiencies in these nutrients, the $\beta$ cells eventually become dysfunctional leading to diabetes.

\section{Adipocytes and cholesterol homeostasis}

The liver is the primary source of synthesized cholesterol, which supplies the body's supplemental needs when dietary cholesterol is deficient. However, the adipocytes from the adipose tissue are mainly responsible for buffering up cholesterol stores to be released into the bloodstream whenever demand exceeds immediate supply from the liver. Adipocytes are unique in their ability to store free or unesterified cholesterol, but they can only do so if they also contain a proportional amount of lipid droplets [37]. ApoB-100, the apolipoprotein found in LDL, inhibits lipolysis in adipocytes, whereas apoB-48, the one found in the chylomicron, does not [38]. Thus, if LDL levels are high, and the chylomicron supply is depleted, fats will accumulate in the adipocytes, and the reason is likely because they are needed to buffer the stored cholesterol.

The free cholesterol is stored in a lipoprotein monolayer encasing the lipid stores, which also uniquely contains proteins involved in protein folding and normally found in the endoplasmic reticulum (ER). Thus, it is becoming apparent that, in adipocytes, the ER forms an intimate relationship with lipid droplets, and a constant exchange between free and esterified cholesterol tightly controls the amount of free cholesterol present in the ER membrane. Ordinarily, there is also a constant exchange between the free cholesterol found in the ER and that in the PM, with exodus from the cell into the serum being triggered by the needs of the body's other tissues.

The adipocytes are also assigned the arduous task of reconstituting cholesterol derived from damaged VLDL remnants, the so-called small dense $\mathrm{LDL}$ that are considered the most damaging in the atherosclerosis process. This task is also normally assumed by the liver as well, but under conditions of excess fructose the liver is overwhelmed and unable to keep up. Adipocytes synthesize large amounts of apoE, which is a necessary catalyst for both the uptake of damaged LDL and the release of the reconstituted cholesterol back into the bloodstream as HDL-C $[39,40]$. The LDL is broken down into lipids, proteins, and cholesterol in the lysosome, and the cholesterol must be refurbished by apoE before it can return to the membrane for absorption into HDL. ApoE, being an antioxidant, is itself especially susceptible to excess glycation and oxidation, which causes it to perform inadequately. Once damaged, apoE can no longer promote transfer of refurbished cholesterol to the PM. The adipocyte then accumulates an excess of cholesterol which needs to be buffered by fats or else it will crystallize and become toxic to the cell. Hence the adipocyte is compelled to become engorged with excess fatty acids. An early study [41], recognizing a unique aspect of adipocytes with respect to LDL uptake, hinted at the idea of AGE'd LDL and the possibility that adipocytes dispose of it. They observed that fat tissue is "a very large cholesterol storage organ". Their studies verified that adipocytes are able to "bind, internalize, and degrade" significant amounts of methylated LDL. Methylation is similar to glycation in that methyl radicals bind to the lysines in ApoB. They observed that there is a "unique physiological function of 
adipocytes to store rather than shun free cholesterol". Most remarkably, they showed in in vitro experiments that an excess of unmethylated LDL in the medium increased the adipocytes' rate of degrading methylated LDL. This suggests that adipocytes are able to selectively degrade and clear damaged LDL.

Another piece of the puzzle is the roles played by calcium and vitamin D. Calcium transport across the ER membrane is crucial for the protein folding step that is required to synthesize viable proteins in the ER [42]. Vitamin D catalyzes calcium transport. Furthermore, the ER membrane is typically depleted in cholesterol compared to the $\mathrm{PM}$, and this gives it greater fluidity to calcium. When cholesterol builds up in the ER, because it is unable to migrate to the PM, the ER becomes stiffer and resistant to calcium transport. This results in dysfunctional protein folding machinery, which may be the primary factor contributing to subsequent cell death. Meanwhile the PM becomes more permeable due to cholesterol depletion, leading initially to excess sodium leakage but ultimately to membrane disintegration and cell death.

\section{In vitro experiments with cholesterol depletion}

A research study involving manipulation of cholesterol in the PM of adipocytes has led to great insight regarding the crucial role cholesterol plays in insulin resistance and the inflammatory response [43]. The authors propose that intracellular cholesterol might serve as a link between fat cell size and adipocyte metabolic activity. In in vitro experiments, fat cells isolated from rat subcutaneous fat tissue were treated with increasing concentrations of methyl- $\beta$-cyclodextrins (M $\beta C D$ ), which extract cholesterol from the PM. The cells responded in a dose-dependent manner by (1) activating genes associated with cholesterol synthesis, uptake, and transport, as would be expected (2), upregulating expression of fatty acid synthase (FAS) and downregulating expression of the insulin-regulated glucose transporter type 4 (GLUT4), activities associated with insulin resistance, and (3) upregulating angiotensin, TNF- $\alpha$, and IL- 6 , all known to be involved in the inflammatory stress response of metabolic syndrome. The authors hypothesize that disorganization of cholesterol-rich caveolae, in which the insulin receptor is located, might play an important role in insulin resistance. It can be concluded that engorged adipocytes in MetS manifest a metabolic profile strikingly parallel with that induced by artificially extracting cholesterol because they too are suffering from cholesterol deficiency in the PM.

\section{Endoplasmic reticulum dysfunction due to cholesterol loading}

We thus hypothesize that adipocytes become stressed upon depletion of cholesterol in their PM, concurrent with an excess of cholesterol in the ER. They are compelled to obtain an increased supply of fatty acids. Thus they must synthesize fatty acids from glucose, in order to be able to safely store the excess free cholesterol that they are unable to transport to the membrane due to damaged apoE.

At the same time, excess cholesterol in the ER leads to a reduced rate of transfer of calcium across its membrane due to a decrease in membrane fluidity. Calcium is essential for chaperone proteins such as binding immunoglobulin protein (BiP) that are involved in protein folding in the ER. Therefore, the cells begin to hoard calcium and vitamin D as compensation.

$\mathrm{BiP}$ has become recognized as a ubiquitous protein found in the ER, and it plays a key role as a chaperone for protein folding to realize the correct tertiary structure for proper functioning [42]. BiP directly binds to growing chains and stimulates ATPase activity to generate energy. When BiP is dysfunctional, misfolded proteins must be disposed of by proteolytic breakdown. The ER lumen where $\mathrm{BiP}$ operates is characterized by a high concentration of free calcium. Likely due to the importance of calcium to BiP function, BiP plays an important role in the storage of the rapidly exchanging calcium pool. When the ER becomes over-supplied with cholesterol, its membrane fluidity is reduced, which interferes with the free flow of calcium across the membrane. This leads to calcium depletion in the lumen and an inability to properly fold proteins being synthesized, and likely will cascade to cell death if the situation is not repaired. A temporary fix is to hoard excess calcium along with excess vitamin $D$ to promote calcium transport. This in turn leads to extreme deficiencies of both calcium and vitamin D in the blood serum.

\section{Macrophages and designer mice - the essential roles of BiP and acyl-CoA - cholesterol acyltransferase}

Cholesterol loaded macrophages (foam cells) are a prominent component of atherosclerotic plaque, and it is well accepted that they contribute significantly to the progression of advanced lesions [44]. Macrophage foam cells are created by the uptake of oxidized LDL, which in turn is actively induced in the artery wall by reaction with nitric oxide and $\mathrm{H}_{2} \mathrm{O}_{2}$ under oxidative conditions [45]. The oxidized LDL enters the lysosome, and the extracted cholesterol is delivered to the PM in just 40 minutes [46] via a mechanism that involves apoE. It is only after excess cholesterol has accumulated in the PM 
that cholesterol is then returned to the cytoplasm and converted via acyl-CoA:cholesterol acyltransferase1 (ACAT1) into cholesterol esters to be stored in accumulating lipid deposits, thus converting the macrophage into a foam cell. The main purpose for extracting cholesterol from damaged LDL is to recycle it into $\mathrm{HDL}-\mathrm{A} 1$, i.e., to achieve reverse cholesterol transport. Interestingly, and most significantly, the rate of cholesterol efflux from the PM to apoA1-HDL is ten times greater from macrophage PMs than from smooth muscle cell PMs.

\section{ACAT is essential to macrophage health}

Over the last few decades, researchers have become increasingly sophisticated in applying techniques that involve designer mice to help unravel the mysteries of atherosclerotic plaque build-up. While these experiments have been very fruitful, they have sometimes led to extremely surprising results. Overall, it seems clear that the macrophages present in large numbers in the atherosclerotic plaque play an extremely important role in repairing damaged cholesterol and delivering it to HDL. Hence, they are intimately involved in reverse cholesterol transport, leading to a beneficial increase in the HDL/LDL ratio. Ill-conceived schemes to develop drugs that interfere with the process of cholesterol uptake by macrophages have resulted in obvious failure [47-49]. But the results are expected if it is taken the point of view that macrophages play a crucial positive role that must be maintained intact. We argue that the plaque build-up is a direct consequence of the steady erosion of the ability of adipocytes and liver cells to participate in reverse cholesterol transport. The impairments that they develop over time are a direct consequence of their chronic exposure to AGE products. Macrophages in the atherosclerotic plaque eventually succumb to a similar fate, but not before they have successfully contributed to refuelling $\mathrm{HDL}$.

Macrophages in plaque actively take up oxidized and acetylated LDL and incorporate the extracted cholesterol into accumulating fatty deposits, and this process requires esterification utilizing the enzyme ACAT1 [50, 51]. It had therefore been hoped that interference with ACAT1 synthesis might reduce plaque build-up. However, results have been dismally disappointing $[47,48]$, with plaque buildup continuing unabated but with increased pathology due to the accumulation of cholesterol crystals.

Hyperlipidemic mice, either apoE-/- or LDL-R-/-, that are also defective in ACAT1 synthesis exhibit severe pathology in the skin due to the massive accumulation of extracellular cholesterol crystals, and die at a young age [49]. Their serum cholesterol is lowered, but at a severe cost. This result clearly indicates that ACAT1 functions normally in the skin and is necessary for the recycling of the cholesterol extracted from damaged LDL back into HDL for eventual delivery to the tissues. Experiments cleverly designed to only introduce ACAT1 deficiency selectively in the macrophages, through marrow transplantation of defective stem cells, resulted in an increased area of atherosclerotic lesions heavily laden with cholesterol crystals, along with an increase in inflammation [47]. Hence, ACAT1 is a necessary enzyme for maintaining relatively healthy plaque, and is part of the solution rather than part of the problem.

\section{Experiments with cholesterol-loaded macrophages}

A set of experiments [52] has demonstrated the close coupling between phospholipid synthesis and cholesterol loading. By exposing macrophages to excess free cholesterol over a period of 4 days, it was shown that initially the cells remained healthy but only by synthesizing extra phospholipids to maintain the proper proportion of cholesterol in the PM. Eventually, their ability to synthesize sufficient phospholipids deteriorated. As a consequence, they became necrotic, with swollen organelles and disrupted membranes. The authors also showed that externally supplied cholesterol-depleted HDL protected the cells from necrosis, by actively taking up the excess cholesterol in the PM.

\section{Excess endoplasmic reticulum calcium leads to apoptosis in macrophages}

In a series of experiments, Li et al. [53] demonstrated compellingly that the accumulation of excess cholesterol in the ER is the trigger for ultimate apoptosis of macrophage foam cells in atherosclerotic lesions, which, in turn, likely contribute to plaque destabilization. The ER membrane achieves its fluidity to transport of small ions like $\mathrm{Na}^{+}$and $\mathrm{Ca}^{2+}$ by being relatively depleted in cholesterol [54]. When the ER is unable to unload its accumulating free cholesterol to the PM, the sarcoplasmic reticulum calcium ATPase SERCA2b, a membrane protein that catalyzes calcium transport, loses function due to decreased conformational freedom. This, in turn, causes a depletion of calcium in the ER lumen. Several proteins involved in orchestrating protein folding, such as BiP, calreticulin, and calnexin, are calcium dependent. Low calcium in the ER thus induces the so-called unfolded protein response (UPR), which essentially cripples the cell and triggers cell death.

We propose that a similar process takes place first in subcutaneous adipocytes, whose apoE has been chronically exposed to AGE debris. Initially the 
adipocytes attempt to compensate by hoarding calcium, but ultimately they succumb and become scavenged by macrophages, which take on the dangerous task of clearing damaged LDL. Macrophages also make their way into the ectopic fatty deposits in the major organs, and there, too, they eventually succumb to glycation and fructation damage and suffer a similar fate. The macrophages in the cardiac arterial wall are the last frontier, forced to take on the task of recycling damaged LDL because the subcutaneous and ectopic fatty tissues are already severely compromised.

\section{Calcium and vitamin D deficiency in the obese}

Calcium and vitamin D deficiency in the obese has been attributed to excessive storage of 25 hydroxyvitamin D in adipose tissue. Zemel et al. [55] claim that low calcium diets promote excess storage of fats in fat cells. They have confirmed in studies with rats as well as through both epidemiological and clinical trial data with humans that high calcium intakes afford protection from obesity. They and others [56] have observed, for obese people, a significant weight loss associated with augmented calcium ingestion, whether through calcium supplements or via dietary adjustments. Calcium obtained from dairy products was particularly effective as compared with other sources of calcium. This is likely because lactose enhances intestinal absorption of calcium [57].

It has been known for some time that patients following gastric bypass surgery suffer from severe vitamin D deficiency. However, it has also been noted that, even before bypass surgery, the morbidly obese are vitamin D deficient [58]. In our view, their abundant oversized adipocytes are hoarding vitamin $\mathrm{D}$ along with calcium because vitamin D catalyzes calcium transport.

\section{Pancreatic $\beta$ cell dysfunction and insulin resistance}

Pancreatic $\beta$ cells are the sole producers of insulin. Beta cells have stringent nutrient requirements before they will release insulin into the bloodstream. In particular, there must be sufficient amounts of cholesterol [59], vitamin D [60], calcium $[59,61]$ and fats [61] available in the cytoplasm, as well as an abundant supply of glucose to fuel the synthesis and release of insulin.

In experiments where the squalene epoxidase inhibitor NB598 was used to selectively impair cholesterol synthesis in mouse pancreatic $\beta$ cells, it was demonstrated that insulin secretion in the presence of glucose was significantly impaired, and that this was largely due to inhibition of voltagegated calcium channels [59]. Both the release of granules stored in membrane-associated releasable vesicles and the refilling of these vesicles were impaired.

Li et al. [62] have demonstrated through experiments with Syt-VII-/-mice that calcium is essential both for insulin transport to the PM in $\beta$ cells and for GLUT4-mediated glucose uptake by skeletal muscles and adipocytes. Syt-VII is a synaptotagmin which serves as a calcium sensor for vesicular traffic, and it mediates secretory granular exocytosis. It regulates both insulin secretion by $\beta$ cells and GLUT4 translocation in insulin-sensitive tissues. Syt-VII-/-mice are glucose intolerant and exhibit significantly less insulin secretion than their control littermates.

We explained above that adipocytes would hoard calcium because their ER is super-saturated in cholesterol. It is evident, however, that serum calcium deficiency will lead to impairment in both insulin release and glucose uptake, as an indirect consequence of adipocyte dysfunction. A characteristic of obesity is excess leptin and suppressed adiponectin signalling. Insufficient adiponectin promotes insulin resistance and the preferential uptake of fats over glucose in the muscle cells [63], and its intentional suppression is likely related to an anticipated impairment in glucose metabolism due to calcium depletion.

This situation is further aggravated by poor calcium absorption in the gut. Fats promote calcium absorption, and dietary fibre interferes with it [64]. So a high-fibre diet, if associated with a stringent low-fat diet may compound the problem of insulin resistance and $\beta$ cell dysfunction.

\section{Lipid rafts, bile salts, and HDL-C}

Epidemiological studies have demonstrated the significant inverse relationship between HDL-C and coronary atherosclerosis. The Framingham Heart Study reported that HDL-C had the strongest inverse relationship to incidence of $C A D$, and that depleted cholesterol in HDL is the best lipid predictor of coronary artery disease for people over 50 years old. HDL-C is also an important predictor for chances of surviving bypass surgery. Foody et al. [65], in a clinical study at the Cleveland Clinic, found that men whose HLD-C was in the lowest quartile had a $33 \%$ reduced chance of survival after 15 years. In general, a $1 \mathrm{mg} / \mathrm{dl}$ increase in HDL-C level is associated with a significant reduction in CAD risk of $2 \%$ in men and of $3 \%$ in women.

The HDL receptor SR-BI plays a critical role in reverse cholesterol transport [66], returning spent lipoproteins to the liver so that they can be recycled. Under normal conditions, the extracted cholesterol is secreted to the gall bladder to be incorporated into bile acids, which promotes the digestion of fats. The refurbished cholesterol is then reintroduced to the tissues along with the fats via the chylomicron. 
This then becomes an important source of high quality cholesterol for the tissues through a "back door" pathway.

It has recently become clear that the PM is a much more complex structure than had previously been thought, and attention has focused on the increasingly accepted idea that so-called lipid rafts, cholesterol and sphingolipid enriched domains, play an important role in the mediation of transport of fatty acids and glucose across the PM through their involvement in a variety of signalling pathways [67].

Given the hypothesis that lipid rafts and caveolae might play a role in cholesterol metabolism in the intestine, researchers at the University of lowa [68] conducted experiments on human small intestine cells to detect the presence of caveolin mRNA. They confirmed its presence and determined that caveolin is associated with detergent-resistant microdomains of cellular membranes, i.e., with lipid rafts. Cholesterol is transported from these caveolae to the ER for esterification and eventual incorporation into the chylomicron. This process likely requires sufficient dietary fat to associate with the esterified cholesterol, to prevent toxicity. Hence, the amount of cholesterol in bile salts has to be kept in check if dietary fats are depleted.

An experiment where human caveolin-1 and caveolin-2 were transfected into hepatocytes in mice showed clearly that caveolins are also heavily involved in bile salt export [69] from the liver. Serum HDL-C was also significantly increased in the transfected mice. It follows logically that a reduction in bile salt export would likely correspond to a reduction in serum $H D L$, i.e., a less favourable profile for atherosclerosis. A low-fat high-fructose diet would lead to a reduction in bile salt export, due to both the decreased need for bile salts to digest fats and the increased load on the liver to synthesize and store sufficient cholesterol to associate with the accumulating fat deposits synthesized from the fructose.

\section{Adipocytes release angiotensin II when stressed}

The depletion of cholesterol in the adipocyte PM leads to sodium leaks [36], and this will result in excess serum sodium and in the activation of the renin-angiotensin system, which in turns causes profound vasoconstriction with a resulting increase in blood pressure. Hypertension is one of the salient features of MetS [1].

Angiotensin II has also been shown to increase the gene expression of inflammatory agents, and decrease adiponectin expression [70]. Since adiponectin increases insulin sensitivity in skeletal muscles, its decrease will promote insulin resistance and a preference for fatty acids over glucose as substrate. This is likely an endocrine action on the part of adipocytes induced to protect skeletal muscles, likely also depleted in PM cholesterol, from potential glycation and oxidative damage to myoglobin as a consequence of excess vulnerability without the protection afforded by cholesterol in lipid rafts.

Angiotensin II has a complex and highly significant effect on apoE expression, sharply decreasing its synthesis in adipocytes, while increasing its expression in peritoneal macrophages [70]. This suggests that the intent is to transfer control of clearance of damaged LDL from adipocytes to macrophages, since the adipocyte can no longer safely execute this function. Through in vitro experiments, it has been demonstrated that oxidant stress sharply downregulates apoE expression in adipocytes in obesity [71].

Angiotensin II, similar to insulin, increases the expression of SR-BI in adipocytes, which promotes the uptake of cholesterol from HDL [72]. Ironically, the adipocyte must extract cholesterol from HDL to supply additional cholesterol to its expanding PM, while simultaneously accumulating excess fats (synthesized from glucose) to allow it to safely store the excess free cholesterol trapped internally in the ER.

\section{Experiments with genetically engineered obese mice}

Genetically manipulated mice have become a valuable resource for uncovering the metabolic effects of obesity and the roles that adiponectin and leptin play in controlling homeostasis. A recent study [73] compared three mouse phenotypes: WT (wild type controls), ob/ob (obese mice with defective leptin synthesis) and transgenic (ob/ob mice engineered to over-express adiponectin). Interestingly, the transgenic mice grew even more obese than the ob/ob mice, but their subcutaneous fat tissues were characterized by both significantly less macrophage penetration and a larger number of fat cells with much smaller average size. The authors hypothesized that, in the case of the ob/ob mice, necrosis due to membrane disruption of engorged adipocytes led to the infiltration of macrophages and subsequent release of inflammatory agents.

The transgenic mice exhibited improved prognosis on a number of indicators of MetS, compared to ob/ob - improved insulin sensitivity, substantially reduced ectopic fat deposition, and lower fasting glucose, triglyceride and FFA levels. However, they clearly were not healthy, in that they behaved as if they were starved. They were very inactive, and unable to maintain body temperature in cold conditions. Furthermore, and most significantly, while they deposited very little visceral fat elsewhere, they exhibited a five-fold increase in pericardial fat deposits. During fasting conditions, 
the transgenic females suffered more readily from hypoglycaemia, indicating an impaired ability of the liver to initiate gluconeogenesis. The buffered fat available to the heart may serve a crucial role in protecting this essential organ from gross nutritional insufficiency under fasting conditions. Notably, pericardial fat deposits have been shown to be highly correlated with atherosclerotic plaque in human studies, and in fact were found to be more highly associated with early development of coronary artery disease than simple anthropometric measures of abdominal obesity [74].

MetS is a syndrome commonly defined as the presence of at least three metabolic indicators among a list of factors associated with increased risk to cardiovascular disease. One of the main objections to the use of the term is the lack of a theory that adequately explains why this particular set of metabolic manifestations dyslipidemia, high blood pressure, excess visceral fat, and impaired glucose metabolism - should coexist. In this paper, we have developed a novel theory which can explain the mechanisms related to MetS pathogenesis, based on the damaging effects of a dietary excess of high-glycemic-index carbohydrates, particularly fructose, in conjunction with an avoidance of dietary cholesterol. We provide support for the theory from several animal studies, involving hamsters, baboons, and rodents, showing a rapid and remarkable adverse effect of fructose on liver function. A crucial piece of the puzzle is the observation that glycated LDL is more susceptible to oxidative damage, including oxidation of the contained cholesteryl esters.

Adipocyte and macrophage dysfunction contribute significantly to the disease process. Both in vitro and animal studies of cholesterol metabolism by these cells reveal that they carry the burden of extracting cholesterol from glycated LDL and transporting it to HDL-A1 via a scavenger mechanism. In the process, they too become damaged, leading eventually to apoptosis and cell death. AGE damage to apoE blocks the movement of cholesterol from the ER to the PM, and excess cholesterol accumulates in the ER. The adipocytes become engorged with fatty deposits to buffer the excess cholesterol, while simultaneously suffering from depleted PM cholesterol. Subsequent defects in the cholesterol supply chain, along with excessive storage of 25-hydroxyvitamin D by the adipocytes, leads to dysfunctional $\beta$ cells in the pancreas along with insulin resistance in the skeletal muscles and other tissues. Distressed adipocytes secrete leptin, angiotensin-II, and cytokines, which orchestrate a major restructuring of muscle metabolism towards favouring fats over glucose, and induce macrophages to invade the adipose tissue to clean up cell debris.
The supply of triglycerides and glucose in the bloodstream must be kept artificially high because widespread insulin resistance interferes with the utilizing of these nutrients for fuel. The depletion of cholesterol in the adipocyte PM leads to sodium leaks, and this will result in excess serum sodium and in the activation of the renin-angiotensin system leading to hypertension. Calcium hoarding by adipocytes and insufficient cholesterol supply likely contribute to impaired insulin synthesis and secretion in pancreatic $\beta$ cells. Finally, due to the problem of ineffective supply from the bloodstream lipid particles, private stashes of fat and cholesterol accumulate ectopically.

In conclusion, we would urge medical practitioners to encourage individuals exhibiting MetS to strongly limit the consumption of dietary fructose [75] and other high-glycemic-index carbohydrates, and to stop discouraging them from consuming foods rich in cholesterol [76].

\section{References}

1. Redon J, Martinez F, Fabia MJ. The metabolic syndrome in hypertension. Arch Med Sci 2009; 5: S320-9.

2. Grundy SM, Brewer BH Jr, Cleeman JI, Smith SC Jr, Lenfant C. Definition of Metabolic Syndrome: Report of the National Heart, Lung, and Blood Institute/American Heart Association Conference on Scientific Issues Related to Definition. Circulation 2004; 109: 433-8.

3. Ferrannini E. Metabolic syndrome: a solution in search of a proble(m. J Clin Endocrinol Metabol 2007; 92: 396-8.

4. Oosting SF, Lefrandt JD, Wolffenbuttel BH, Sleijfer DT, Gietema JA. The metabolic syndrome in cancer survivors. Lancet Oncol 2010; 11: 193-203.

5. Jeppesen J, Hein HO, Suadicani P, Gynterberg F. Low triglycerides-high high-density lipoprotein cholesterol and risk of ischemic heart disease. Arch Intern Med 2001; 16: 361-6.

6. Onat A, Hergen C, Sari I, Türkmen S, Can G, Sansoy V. Dyslipidemic hypertension: distinctive features and cardiovascular risk in a prospective population-based study. Am J Hypertens 2005; 18: 409-16.

7. Dekker JM, Girman C, Rhodes T, et al. Metabolic syndrome and 10-year cardiovascular disease risk in the Hoorn Study. Circulation 2005; 112: 666-73.

8. Ford ESMM. Prevalence of the Metabolic Syndrome Defined by the International Diabetes Federation Among Adults in the U.S. Diabetes Care 2005; 28: 2745-9.

9. Forsythe CE, Phinney SD, Fernandez ML, et al. Comparison of low fat and low carbohydrate diets on circulating fatty acid composition and markers of inflammation. Lipids 2008; 43: 65-77.

10. Volek JS, Phinney SD, Forsythe CE, et al. Carbohydrate restriction has a more favorable impact on the metabolic syndrome than a low fat diet. Lipids 2009; 44: 297-309.

11. Höfer J, Niebauer J. Cardiovascular risk factors: lipids and lifestyle changes. Arch Med Sci 2007; 3: S69-S73.

12. Kaitosaari T, Rönnemaa T, Viikari J, Leino A, Jokinen E, Simell O. Low-density lipoprotein (LDL) particle size in healthy prepubertal children: the STRIP study. Acta Paediatr 2006; 95: 1668-73.

13. Reaven GM. Pathophysiology of insulin resistance in human disease. Physiol Rev 1995; 75: 473-86. 
14. Castelli WP, Garrison RJ, Wilson PW, Abbott RD, Kalousdian S, Kannel WB. Incidence of coronary heart disease and lipoprotein cholesterol levels. The Framingham Study. JAMA 1986; 256: 2835-8.

15. Levi B, Werman MJ. Long-term fructose consumption accelerates glycation and several age-related variables in male rats. J Nutr 1998; 128: 1442-9.

16. Dai S, McNeill JH. Fructose-induced hypertension in rats is concentration-and duration-dependent. J Pharmacol Toxicol Methods 1995; 33: 101-7.

17. Lin Y, Tseng Y, Chang K. Aminoguanidine prevents fructose-induced arterial stiffening in Wistar rats: aortic impedance analysis. Exp Biol Med 2004; 229: 1038-45.

18. Tran LT, Yuen VG, McNeill JH. The fructose-fed rat: a review on the mechanisms of fructose-induced insulin resistance and hypertension. Mol Cell Biochem 2009; 332: 145-59.

19. Le, Ith M, Kreis R, Faeh D, Bortolotti M, Tran C, Boesch C, Tappy L. Fructose overconsumption causes dyslipidemia and ectopic lipid deposition in healthy subjects with and without a family history of type 2 diabetes. Am J Clin Nutr 2009; 89: 1760-5.

20. Pasaoglu H, Sancak B, Bukan N. Lipid peroxidation and resistance to oxidation in patients with type 2 diabetes mellitus. Tohoku J Exp Med 2004; 203: 211-8.

21. Kritchevsky D, Davidson LM, Shapiro IL, et al. Lipid metabolism and experimental atherosclerosis in baboons: influence of cholesterol-free, semi-synthetic diets. Am J Clin Nutr 1974; 27: 29-50.

22. Taghibiglou C, Carpentier A, Van Iderstine SC, Chen B, Rudy D, Aiton A, Lewis GF, Adeli K. Mechanisms of hepatic very low density lipoprotein overproduction in insulin resistance. Evidence for enhanced lipoprotein assembly, reduced intracellular $A p o B$ degradation, and increased microsomal triglyceride transfer protein in a fructose-fed hamster model. J Biol Chem 2000; 275: 8416-25. Erratum in: J Biol Chem 2001; 276: 20804.

23. Scoppola A, Testa G, Frontoni S, Maddaloni E, Gambardella S, Menzinger G, Lala A. Effects of insulin on cholesterol synthesis in type II diabetes patients. Diabetes Care 1995; 18: $1362-9$.

24. Gylling $H$, Hallikainen M, Pihlajamaki J, Simonen $P$, Kuusisto J, Laakso M, Miettinen TA. Insulin sensitivity regulates cholesterol metabolism to a greater extent than obesity. Lessons from the METSIM study. J Lipid Res 2010; 51: 2422-7.

25. Angel A, Farkas J. Regulation of cholesterol storage in adipose tissue. J Lipid Res 1974; 15: 491-9.

26. Suárez G, Rajaram R, Oronsky AL, Gawinowicz MA. Nonenzymatic glycation of bovine serum albumin by fructose (fructation). Comparison with the Maillard reaction initiated by glucose. J Biol Chem 1989; 264: 3674-9.

27. Kikuchi S, Shinpo K, Takeuchi M, Yamagishi S, Makita Z, Sasaki N, Tashiro K. Glycation - a sweet tempter for neuronal death. Brain Res Brain Res Rev 2003; 41: 306-23.

28. McPherson JD, Shilton BH, Walton DJ. Role of fructose in glycation and cross-linking of proteins. Biochemistry 1988; 27: 1901-7.

29. Brownlee M, Cerami A, Vlassara H. Advanced glycosylation end products in tissue and the biochemical basis of diabetic complications. N Engl J Med 1988; 318: 1315-21.

30. Zimmermann R, Panzenböck U, Wintersperger A, et al. Lipoprotein lipase mediates the uptake of glycated LDL in fibroblasts, endothelial cells, and macrophages. Diabetes 2001; 50: 1643-53.

31. Ravandi A, Kuksis A, Shaikh NA. Glucosylated glycerophospho-ethanolamines are the major Idl glycation products and increase LDL susceptibility to oxidation: evidence of their presence in atherosclerotic lesions. Arterioscler Thromb Vasc Biol 2000; 20: 467-77.

32. Olson RE. Discovery of the lipoproteins, their role in fat transport and their significance as risk factors. J Nutr 1998; 128: 439S-43S.

33. Lakshmanan MR, Maten MV, Muesing RA, O'Looney P, Vahouny GV. Role of high density lipoproteins in regulation of hepatic fatty acid synthesis by chylomicron and very low density lipoprotein remnants. J Biol Chem 1983; 258: 4746-9.

34. Li YM, Dickson DW. Enhanced binding of advanced glycation endproducts (AGE) by the ApoE4 isoform links the mechanism of plaque deposition in Alzheimer's disease. Neurosci Lett 1997; 226: 155-8.

35. Cinti S, Mitchell G, Barbatelli G, et al. Adipocyte death defines macrophage localization and function in adipose tissue of obese mice and humans. J Lipid Res 2005; 46: 2347-55.

36. Haines TH. Do sterols reduce proton and sodium leaks through lipid bilayers? Prog Lipid Res 2001; 40: 299-324.

37. Prattes S, Horl G, Hammer A, Blaschitz A, Graier WF, Sattler W, Zechner R, Steyrer E. Intracellular distribution and mobilization of unesterified cholesterol in adipocytes: triglyceride droplets are surrounded by cholesterol-rich ERlike surface layer structures. J Cell Sci 2000; 113: 2977-89.

38. Skogsberg J, Dicker A, Ryden M, et al. ApoB100-LDL acts as a metabolic signal from liver to peripheral fat causing inhibition of lipolysis in adipocytes. PLoS One 2008; 3 : e3771.

39. Huang ZH, Reardon CA, Mazzone T. Endogenous ApoE expression modulates adipocyte triglyceride content and turnover. Diabetes 2006; 55: 3394-402.

40. Zhang SH, Reddick RL, Piedrahita JA, Maeda N. Spontaneous hypercholesterolemia and arterial lesions in mice lacking apolipoprotein E. Science 1992; 258: 468-71.

41. Fong BS, Rodrigues PO, Angel A. Characterization of low density lipoprotein binding to human adipocytes and adipocyte membranes. J Biol Chem 1984; 259: 10168-74.

42. Lievremont JP, Rizzuto R, Hendershot L, Meldolesi J. BiP, a major chaperone protein of the endoplasmic reticulum lumen, plays a direct and important role in the storage of the rapidly exchanging pool of Ca2+. J Biol Chem 1997; 272: 30873-9.

43. Le Lay S, Krief S, Farnier C, et al. Cholesterol, a cell sizedependent signal that regulates glucose metabolism and gene expression in adipocytes. J Biol Chem 2001; 276: 16904-10.

44. Smith JD, Trogan E, Ginsberg M, Grigaux C, Tian J, Miyata M. Decreased atherosclerosis in mice deficient in both macrophage colony-stimulating factor (op) and apolipoprotein E. Proc Natl Acad Sci U S A 1995; 92: 8264-8.

45. Mertens A, Holvoet P. Oxidized LDL and HDL: antagonists in atherothrombosis. FASEB J 2001; 15: 2073-84.

46. Liscum L, Underwood KW. Intracellular cholesterol transport and compartmentation. J Biol Chem 1995; 270: 15443-6.

47. Fazio S, Major AS, Swift LL, et al. Increased atherosclerosis in LDL receptor-null mice lacking ACAT1 in macrophages. J Clin Invest 2001; 107: 163-71.

48. Fazio S, Linton M. Failure of ACAT inhibition to retard atherosclerosis. N Engl J Med 2006; 354: 1307-9.

49. Accad M, Smith SJ, Newland DL, et al. Massive xanthomatosis and altered composition of atherosclerotic lesions in hyperlipidemic mice lacking acyl CoA:cholesterol acyltransferase 1. J Clin Invest 2000; 105: 711-9. 
50. Akiba S, Yoneda Y, Ohno S, Nemoto M, Sato T. Oxidized LDL activates phospholipase A2 to supply fatty acids required for cholesterol esterification. J Lipid Res 2003; 44: 1676-85.

51. Brown MS, Ho YK, Goldstein JL. The cholesteryl ester cycle in macrophage foam cells. Continual hydrolysis and reesterification of cytoplasmic cholesteryl esters. J Biol Chem 1980; 255: 9344-52.

52. Tabas I, Marathe S, Keesler GA, Beatini N, Shiratori Y. Evidence that the initial up-regulation of phosphatidylcholine biosynthesis in free cholesterol-loaded macrophages is an adaptive response that prevents cholesterol-induced cellular necrosis. Proposed role of an eventual failure of this response in foam cell necrosis in advanced atherosclerosis. J Biol Chem 1996; 271: 22773-81.

53. Li Y, Ge M, Ciani L, et al. Enrichment of endoplasmic reticulum with cholesterol inhibits sarcoplasmicendoplasmic reticulum calcium ATPase- 2 b activity in parallel with increased order of membrane lipids: implications for depletion of endoplasmic reticulum calcium stores and apoptosis in cholesterol-loaded macrophages. J Biol Chem 2004; 279: 37030-9.

54. Lange Y, Steck TL. Quantitation of the pool of cholesterol associated with acylCoA:cholesterol acyltransferase in human fibroblasts. J Biol Chem 1997; 272: 13103-8.

55. Zemel MB, Miller SL. Dietary calcium and dairy modulation of adiposity and obesity risk. Nutr Rev 2004; 62: 125-31.

56. Schrager S. Dietary calcium intake and obesity. J Am Board Fam Pract 2005; 18: 205-10.

57. Au WY, Raisz LG. Restoration of parathyroid res ponsiveness in vitamin D-deficient rats by parenteral calcium or dietary lactose. J Clin Invest 1967; 46: 1572-8.

58. Buffington C, Walker B, Cowan Jr G, Scruggs D. Vitamin D deficiency in the morbidly obese. Obes Surg 1993; 3: 421-4

59. Xia F, Xie L, Mihic A, et al. Inhibition of cholesterol biosynthesis impairs insulin secretion and voltage-gated calcium channel function in pancreatic beta-cells. Endocrinology 2008; 149: 5136-45.

60. Norman AW, Frankel JB, Heldt AM, Grodsky GM. Vitamin D deficiency inhibits pancreatic secretion of insulin. Science 1980; 209: 823-5.

61. Corkey BE, Deeney JT, Yaney GC, Tornheim K, Prentki M. The role of long-chain fatty acyl-CoA esters in beta-cell signal transduction. J Nutr 2000; 130: 299S-304S.

62. Li Y, Wang P, Xu J, Gorelick F, Yamazaki H, Andrews N, Desir GV. Regulation of insulin secretion and GLUT4 traficking by the calcium sensor synaptotagmin VII. Biochem Biophys Res Commun 2007; 362: 658-64.

63. Maeda N, Shimomura I, Kishida K, et al. Diet-induced insulin resistance in mice lacking adiponectin/ACRP30. Nat Med 2002; 8: 731-7.

64. Wolf RL, Cauley JA, Baker CE, et al. Factors associated with calcium absorption efficiency in pre- and perimenopausal women. Am J Clin Nutr 2000; 72: 466-71.

65. Foody JM, Ferdinand FD, Pearce GL, Lytle BW, Cosgrove DM, Sprecher DL. HDL cholesterol level predicts survival in men after coronary artery bypass graft surgery: 20-year experience from The Cleveland Clinic Foundation. Circulation 2000; 102: III90-III94.

66. van der Velde AE, Groen AK. Shifting gears: liver SR-BI drives reverse cholesterol transport in macrophages. J Clin Invest 2005; 115: 2699-701.

67. Michel V, Bakovic M. Lipid rafts in health and disease. Bio Cell 2007; 99: 129-40.

68. Field FJ, Born E, Murthy S, Mathur SN. Caveolin is present in intestinal cells: role in cholesterol trafficking? J Lipid Res 1998; 39: 1938-50.
69. Moreno M, Molina H, Amigo L, Zanlungo S, Arrese M, Rigotti A, Miquel JF. Hepatic overexpression of caveolins increases bile salt secretion in mice. Hepatology 2003; 38: 1477-88.

70. Rao P, Huang ZH, Mazzone T. Angiotensin II regulates adipocyte apolipoprotein E expression. J Clin Endocrino Metab 2007; 92: 4366-72.

71. Espiritu DJ, Mazzone T. Oxidative stress regulates adipocyte apolipoprotein e and suppresses its expression in obesity. Diabetes 2008; 57: 2992-8.

72. Yvan-Charvet L, Bobard A, Bossard $P$, et al. In vivo evidence for a role of adipose tissue SR-BI in the nutritional and hormonal regulation of adiposity and cholesterol homeostasis. Arterioscler Thromb Vasc Biol 2007; 27: 1340-5.

73. Kim J, van de Wall E, Laplante $M$, et al. Obesity-associated improvements in metabolic profile through expansion of adipose tissue. J Clin Invest 2007; 117: 2621-37.

74. Konishi M, Sugiyama S, Sugamura K, et al. Association of pericardial fat accumulation rather than abdominal obesity with coronary atherosclerotic plaque formation in patients with suspected coronary artery disease. Atherosclerosis 2010; 209: 573-8.

75. Ferder L, Ferder MD, Inserra F. The role of high-fructose corn syrup in metabolic syndrome and hypertension. Curr Hypertens Rep 2010; 12: 105-12.

76. Wainwright G, Mascitelli L, Goldstein MR. Cholesterol lowering therapies and membrane cholesterol. Stable plaque at the expense of unstable membranes? Arch Med Sci 2009; 5: 289-95. 